\title{
What the Tortoise Said to Achilles: Lewis Carroll's paradox in terms of Hilbert arithmetic
}

\author{
Vasi Penchev, vasildinev@gmail.com \\ Bulgarian Academy of Sciences: institute of Philosophy and Sociology: \\ Dept. of Philosophy of Science
}

Abstract. Lewis Carroll, both logician and writer, suggested a logical paradox containing furthermore two connotations (connotations or metaphors are inherent in literature rather than in mathematics or logics). The paradox itself refers to implication demonstrating that an intermediate implication can be always inserted in an implication therefore postponing its ultimate conclusion for the next step and those insertions can be iteratively and indefinitely added ad lib, as if ad infinitum. Both connotations clear up links due to the shared formal structure with other well-known mathematical observations: (1) the paradox of Achilles and the Turtle; (2) the transitivity of the relation of equality. Analogically to (1), one can juxtapose the paradox of the Liar (for Lewis Carroll's paradox) and that of the arrow (for "Achilles and the Turtle"), i.e. a logical paradox, on the one hand, and an aporia of motion, on the other hand, suggesting a shared formal structure of both, which can be called "ontological", on which basis "motion" studied by physics and "conclusion" studied by logic can be unified being able to bridge logic and physics philosophically in a Hegelian manner: even more, the bridge can be continued to mathematics in virtue of (2), which forces the equality (for its property of transitivity) of any two quantities to be postponed analogically ad lib and ad infinitum. The paper shows that Hilbert arithmetic underlies naturally Lewis Carroll's paradox admitting at least three interpretations linked to each other by it: mathematical, physical and logical. Thus, it can be considered as both generalization and solution of his paradox therefore naturally unifying the completeness of quantum mechanics (i.e. the absence of hidden variables) and eventual completeness of mathematics as the same and isomorphic to the completeness of propositional logic in relation to set theory as a first-order logic (in the sense of Gödel (1930)'s completeness theorems).

Keywords: equality, Lewis Carroll's paradox, Liar's paradox, paradox of the arrow, "Achilles and the Turtle", Hilbert arithmetics, qubit Hilbert space

\section{LEWIS CARROLL'S PARADOXES IN ORIGINAL}

Lewis Carroll published in 1994 and in 1995 correspondingly in Mind two concise notices now known (or even famous) as Carroll's "Logical paradox" (or "Barber Shop paradox") and "What the Tortoise said to Achilles". The latter is the main subject of discussion in the present paper, however the former will be interpreted accidently following the connotations of the latter therefore allowing for their additional elucidation (once both had been suggested by the same author almost simultaneously).

The reason for the interest to them now consists in the realized option to be understood in terms of Hilbert arithmetic thus eventually demonstrating that it is able to resolve alleged contradictions between (propositional) logic, (Peano) arithmetic, and (ZFC) set theory as far as all they are necessary for the foundations of mathematics. In turn, Hilbert arithmetic can be considered as a reading of the separable complex Hilbert space utilized by quantum mechanics, 
designated especially to use the "solution of nature" to resolve the problems in the foundations of mathematics ${ }^{1}$

The main immediate idea of Hilbert arithmetic (which can be exemplified by "What the Tortoise said to Achilles", figuratively speaking, after the Tortoise had been "studied" it) consists in the option for the contradiction of logic, arithmetic, and set theory to be reconciled relating each of them to a certain substructure of the same structure, furthermore isomorphic to the separable complex Hilbert space (under a few technical conditions). Then, "What the Tortoise said to Achilles" may mean the essential properties of those three bases, "whales of mathematics", however in such a way that they represent alternative, but absolutely consistent aspects of its foundation.

Lewis Carroll suggested two quite different logical paradoxes very soon one after another, therefore eventually hinting that they share the same problem though formulated so differently at first glance. Now, that problem can be formulated (meaning also Hilbert arithmetic as a background) as the contradiction of propositional logic interpreted once traditionally, i.e. as the rules of conclusion after which any logical proposition is a well-ordered sequence, on the one hand, or interpreted as an algebraic structure such as Boolean algebra, in which the ordering is suspended fundamentally in virtue of both double commutativity and perfect symmetry of conjunction and disjunction, on the other hand.

One can notice that quantum mechanics would been forced about two or three decades later to reconcile analogically (and as this will be demonstrated further: even mathematically isomorphically) any well-ordering after measurement with its coherent counterpart before measurement definitively deprived of any well-ordering (e.g. in virtue of the theorems about the absence of hidden variables in quantum mechanics: Neumann 1932; Kochen, Specker 1967). Just the separable complex Hilbert space inherently supplied by the property of unitarity is able to to reconcile and unify Heisenberg's (well-ordered) matrix mechanics and Schrödinger (commutative ${ }^{2}$ ) ondulatory mechanics.

Projected backwards, to Carroll's paradoxes, that property of unitarity can be interpreted as "logical unitarity" able to resolve what the English writer, mathematician and logician Charles Lutwidge Dodgson (known as Lewis Carroll) had observed as a contradiction. Thus, logical unitarity is to mean the identification of the traditional propositional logic of human conclusion, and the contemporary Boolean logical calculation (e.g. in the foundations of computer science).

\footnotetext{
${ }^{1}$ An approach, which can be called figuratively "cognitive bionics" since bionics is defined to be a new science predetermined to utilize relevant "solutions of nature" to resolve problems in technics, and human cognition and can be also interpreted as a branch of techniques in a wider sense.

${ }^{2}$ The (Hermiian) operators are not commutative in general in both quantum and ondulatory mechanics, which true statement is often abbreviated to: "Quantum mechanics is non-commutative", a statement being true only after that elucidation. However, one means here the commutativity of the additive members of any wave function unlike the well-ordering of the components of any vector therefore representing the proper mathematical sense of unitarity (and which can be visualized by the rotation in complex plane about its beginning and conserving the length of the vectors).
} 
One can describe so, preliminarily and briefly the "spectacles", which will be put for the interpretation of Carroll's paradoxes; however, they should be initially represented literally, in original:

"A Logical Paradox" (Carroll 1894) suggests the following problem as it was formulated by Carroll himself

"There are two Propositions, A and B. It is given that:

(1) If $\mathrm{C}$ is true, then, if $\mathrm{A}$ is true, $\mathrm{B}$ is not true;

(2) If $A$ is true, $B$ is true.

The question is, can C be true?" (Carroll 1894: 438).

Obviously, C can be true since its premise in (1) is false in virtue of (2). Thus, it is not a paradox, but a logical mistake if it is involved in a "proof" utilizing reductio ad absurdum as in the dialogue suggested by Lewis Carroll.

However, the contemporary "obvious" conclusion is crucially influenced by the interpretation of the theory of syllogisms as Boolean algebra ${ }^{3}$.

If one translates the structure of the alleged paradox into syllogisms, the trouble probably meant by Carroll would be:

(1) The negation of the proposition that A implies B implies C (the comma is interpreted as "conjunction");

(2) A implies B.

Then, $\mathrm{C}$ can be both true and false since the premise of the implication, in italic in (2), is not stated by (2).

Also:

(1') The proposition that B implies A implies C (comma is interpreted as "disjunction");

(2') A implies B.

Again, $\mathrm{C}$ can be both true and false since the premise of the implication, in italic in (2), is not stated by (2).

In other words, $\mathrm{C}$ can never be true only if the implication in italic is interpreted (misleadingly) as logical equivalence and an eventual proof involving it into reductio ad absurdum would hold (as in the text of Lewis Carroll).

However, one can notice that unitarity in quantum mechanics (and eventually, the suggested now "logical unitarity") is forced to make just this "logical fallacy", for example, as by identifying the reversible coherent state before measurement and the irreversible (being well-ordered) state after measurement.

In fact, the same logical fallacy is postulated in set theory by the axiom of choice and its provable equivalency with the well-ordering "theorem". Indeed, one may state that both alternatives are equivalent as to a "choice", but not, as to an "ordering" (however both possible orderings are equivalent to each other). ${ }^{4}$

${ }^{3}$ Gillon (1950) reflected on it from the viewpoint of "contraposition" or modus tollens.

${ }^{4}$ One can trace back that "logical fallacy" even to "Hume's problem of induction" and its link to "What the Tortoise said to Achilles" whether explicitly (e.g. Otero 2008; Howson 2000; Belkind 2018) or implicitly and more often (e.g. Jackson 2019; Lange 2011; Weintraub 2008; Tucker 2009; Lipton 2002, a 
So, a possible verdict to "A Logical Paradox" suggested by Lewis Carroll in 1994 is in turn paradoxical: though it is not a real logical paradox (relying on a trivial mistake), its philosophic sense is essential as a presentiment (or "foreshock") of the huge cognitive earthquake constituted quantum mechanics; and this can be confirmed by the specific propositions by which the alleged logical paradox is exemplified:

"The reader will see that if, in these two Propositions, we replace the letters A, B, C by the names Allen, Brown, Carr, and the words 'true' and 'not true' by the words 'out' and 'in' we get:

(1) If Carr is out, then, if Allen is out, Brown is in;

(2) If Allen is out, Brown is out" (Carroll 1894: 438).

So, the corresponding exemplifying question is whether "Carr be out" is possible.

The obvious allusion should be directed to entanglement able to restrict "Carr's degree of freedom" having been a quantum entity (a "quantum barber") therefore limitable to be within the "quantum barbershop" necessarily.

However, that allusion is only a metaphor. The problem eventually underlying it is: how unitarity (i.e. the usual unitarity means by quantum mechanics) is mathematically linked to entanglement; how one can translate entanglement into the language of logic by means of logical unitarity. The discussion of that problem is postponed until Section VI devoted to the logical interpretation of the solution of quantum mechanics.

Anyway, a few preliminary notices about the relevance of Carroll's "logical paradox" to the tension of the traditional and algebraic interpretations of propositional logic are appropriate here. Not paying attention to that tension is usual, referring to their equivalence in the framework of Boolean algebra. However, the process of human conclusion meant to be modelled by traditional logic is always well-ordered, obeying to the imperative requirement for any stage and substage to be meaningful. Thus, it is represented as a well-ordered series of implications, since their order is single and starting from certain premises whether preliminarily justified or granted to be obvious as "axioms".

On the contrary, the calculation of any Boolean expression e.g. modelled by a computer calculation is "meaningless" as far as it need only obey the laws of Boolean algebra just an

review of Howson's book (2000); Lantin 1998; Weintraub 1995; Jacobson 1987); one can mean also Hume's problem of induction implicitly, but Carroll's paper explicitly (e.g. Schueler 1995):

Indeed, if one paraphrases it in the present context, it refers to the relation of the axiom of induction (stating that the possible observations are always necessarily a natural number and thus, finite), and the axiom of infinity (stating that the conclusion of all observations relates to an infinite set and thus, the conclusion does not follow from the premises). Even more, Hume's problem of induction means the same interpretation in terms of propositional logic as Carroll's paradox rather than only the same formal structure relatable also to the Gödel incompleteness theorems (e.g.). The present context will suggest another link (rather implicitly) to Hume's problem of induction mediated sequentially by Kant's consideration of it, his doctrine of transcendentalism, and the interpretation of the latter by "scientific transcendentalism" as here.

${ }^{5}$ For example according to Burks and Copi (1950: 219), "Carroll's puzzle is not a genuine logical paradox of the type of the Russell, the Burali-Forti, the Richard, or the Grelling, but is more akin to the so-called paradoxes of material and strict implication". 
arithmetical calculation obeying the corresponding laws and unlike human thinking always admitting its realization and thus forcing a well-ordering analogical or identical to that of time.

Nonetheless, Boolean algebra guarantees a class of expressions, equivalent to any certain chain of conclusions and sharing the same result after each corresponding calculation (including that determined by the chain of conclusions). The difference consists in the interpretation meaning each step of human conclusion, which allows for the check and control of the process of thought.

As to a computer calculation, the same check and control is done preliminarily and established as a software program is to be accomplished. So, only the ultimate result of the calculation (whether logical or arithmetic) need be meaningful. The corresponding software program is to mean any possible course of calculation guaranteeing for the result to be "meaningful" therefore determining the class of those courses as "meaningful".

Then, one can define "logical unitarity" as the mapping of that class of calculations admissible by the corresponding software program into a class of well-ordered chains of implications (containing at least one element necessarily) and accessible to a human mind. Carroll's "logical paradox" is to be related to logical unirarity as follows. It makes sense only if one can allow that logical unitarity can be violated though itself does not manage to do this since it is a logical fallacy in both cases: as a Boolean calculation and as a series of implications.

However, one can admit that the next attempt undertaken by Lewis Carroll, namely "What Tortoise said to Achilles" is to be realized as a paradox able to demonstrate a violation of logical unitarity though involving an infinite series of implications ${ }^{6}$. The ultimate result seems to be just so unattainable as Achilles to overtake the Tortoise in the famous ancient aporia of Zeno:

Though "ACHILLES had overtaken the Tortoise" (p. 278), she or he suggested him an equivalent (to Zeno's "Achilles and the Tortoise") logical problem preventing any logical implication adding again and again, ad infinitum the same implication as a new necessary condition for itself therefore remoting every time the conclusion to remain still and still unattainable. Here is how Lewis Carroll himself represented the arguments of the insidious Tortoise wanting a revenge over Achilles for his victory in the race, but in a logical way:

“(A) Things that are equal to the same are equal to each other.

(B) The two sides of this Triangle are things that are equal to the same.

(Z) The two sides of this Triangle are equal to each other" (p. 278).

"And if some reader had not yet accepted A and B as true, he might still accept the sequence as a valid one, I suppose?" (p. 278) the subtle Tortoise said, and the sincere Achilles responded:

"No doubt such a reader might exist. He might say 'I accept as true the Hypothetical Proposition that, if A and B be true, $\mathrm{Z}$ must be true; but, I don't accept A and B as true'." (p. 279).

Then, the crafty Tortoise involved again the same logical step:

" Let's call it C," said the Tortoise." - but you don't accept:

${ }^{6}$ For example, Diamond and Kaul (2010) suggest a very curious interpretation in terms of the usual human experience (namely, to clinical trials in terms of individuals versus those of groups). 
(C) If A and B are true, $Z$ must be true" (p. 279)

"(A) Things that are equal to the same are equal to each other.

(B) The two sides of this Triangle are things that are equal to the same.

(C) If $\mathrm{A}$ and $\mathrm{B}$ are true, $\mathrm{Z}$ must be true.

(Z) The two sides of this Triangle are equal to each other" (p. 279)

Well, the perfidious Tortoise continued to knit her or his underhand logical intrigue though one cannot accept all propositions " $\mathrm{A}$ ", "B", and "C":

"(D) If $A$ and $B$ and $C$ are true, $Z$ must be true" (p. 279).

"(E) If $A$ and $B$ and $C$ and $D$ are true, $Z$ must be true. Until I've granted that, of course I needn't grant Z. So it's quite a necessary step, you see?"

"I see," said Achilles; and there was a touch of sadness in his tone" (p. 280).

When the narrator would return to the same spot some months afterwards, he saw that "Achilles was still seated on the back of the much-enduring Tortoise, and was writing in his note-book, which appeared to be nearly full. The Tortoise was saying 'Have you got that last step written down?"' (p. 280).

Obviously, the tenet can be repeated ad infinitum, so Achille should be there until now writing and writing the next step implied necessarily by the last one.

One may formulate "What the Tortoise said to Achilles" concisely and symbolically:

$(A \rightarrow C) \rightarrow\{[A \wedge(A \rightarrow C)] \rightarrow C\}$ (or, " $(A \rightarrow C) \rightarrow\{[A \rightarrow(A \rightarrow C)] \rightarrow C\}$ ") and so on again and again ad infinitum: that process of an intermediate implication again and again postpones the conclusion $\mathrm{C}$ not be able to be ended ever.

One might easily, but facetiously interpret this as if "What AI said to all human beings" would be a response to the "offensive" Turing test "whether AI can think" therefore proving that as if the human being utilizing syllogisms successive over time cannot think in fact".

So, if one reckons with Lewis Carroll's intention of the two successive paradoxes to demonstrate an eventual violation of logical unitarity, then, only the latter attempt, "What the Tortoise said to Achilles" manages to do this correctly. The essential difference to the former one is that it involves an infinite, though implicit and iterative series of derivative implications therefore creating a logical equivalent of Zeno's paradox. On the contrary, his previous "logical paradox" not introducing infinity, though eventually meaning as an analogical idea, but without it, i.e. the ostensible violation of the symmetry of "logical unitarity" (as it is meant above), seems not to manage just for restricting only to a finite counterexample.

Logical unitarity can be interpreted immediately in a philosophical way, very considerable and important nowadays, namely as the problematic fundamental mismatch of AI and human intellect. The group of "human chauvinists" suggests that (1) they are fundamentally different from each other, and (2) human intellect superiors AI necessarily and initially, e.g., in virtue of the fact that the former has been created by the latter.

\footnotetext{
${ }^{7}$ For example, Fumerton (2015) suggests an "internalist" interpretation, after which he justifies equally well two opposite conclusions without being able to choose between them; also commented by Wright (2015).
} 
However, Lewis Carroll's "What the Tortoise said to Achilles" means rather the opposite alternative: (1) they are different from each other and this can be made clear involving an infinite series of derivative implications therefore exemplifying a violation of logical unitarity (2) however, AI is able to surpass human intellect in that situation as far as the latter runs as a process in time: thus needing an "infinite time" to accomplish an "infinite series of implications"; on the contrary, AI is not bound by permanently "making sense", so a jumplike transition is quite natural for it.

In fact, human intellect is also able to temporarily cease that constant reflection of "making sense" by insights as if too surprising just being "leaplike". However, propositional logic does not include any rule referring to insights or to any sudden jump in conclusion interpreting it as a fallacy. Nobody knows how the "jump" due to a logical mistake should be distinguished from that of an absolutely justified creative insight in a non-probabilistic mathematical method ${ }^{8}$, though being especially valuable in human intellect.

Furthermore, one can notice a very instructive contradiction about the interpretation of AI (supposedly needing the capability of insight in definition) based on the contemporary binary computers necessarily being "Turing machines" and thus able only to finite calculations. Indeed, they are created after human beings have been reflected on propositional logic fundamentally incapable to represent any insight (as the cunning Tortoise forces Achilles to "be logical") .

Nonetheless, all contemporary computers as Turing machines (and thus incapable to reflect on their work, e.g. as in "Halteproblem") are constructed to do Boolean operations rather than human syllogisms (though being equivalent to the former ones). Thus, they are released from the permanent human necessity of "making sense" by reflecting from the metalevel: a Turing machine (i.e. a contemporary computer) is able to represent any insight as a jump-like proposition fundamentally unrepresentable as a finite series of syllogisms remaining within the scope of Boolean algebra but out of the traditional propositional logic of syllogisms. Indeed, it is able to represent it, but it does not make it by itself, but only if this jump is described exhaustively in its software program (and thus, representable by an infinite series of syllogisms, equivalent to the logical jump by itself, but cut-eliminable to a single one following Gentzen).

However, if one involves the concept of quantum computer as a generalization of "Turing machine", able to make "infinite calculations" and thus, equivalent to leaplike conclusions, the pathway to the machine modeling of human insights is open. One can notice (Penchev 2021 November 18) more or less metaphorically that the Self, or the conservation of the Self corresponds, to the "subjective time" (but correlating directly with "objective time" of mind, and by it, to that of the world). Any insight needs to "stop the Self" (or the "continuous flow of

\footnotetext{
${ }^{8}$ One can formulate an "insight problem" as to human intellect and analogical to the "Halting problem of Turing machine": one cannot know in advance whether a sudden jump in a chain of logical conclusions is a fallacy, or on the contrary, an insight eventually justifiable further, by restoring a possible logical chain leading to it in a necessary way. The suggested analogy to the Halting problem is not accidental, but essential and underliable by a formal mathematical structure originating from Hilbert arithmetic, and more precisely, from the eventual and idempotent transition from a Peano arithmetic and its dual counterpart.
} 
consciousness" for the logical leap to be accomplishable) to be able to appear, therefore surprising the consciousness necessarily when the Self is "switched on" again.

\section{THE LOGICAL ESSENSE OF THE PARADOXES}

Though the logical, mathematical, physical or at all scientific paradoxes mean an exactly determined formal structure, it is extractable almost always from a short text in a natural language (e.g. ancient Greek, English, etc.) and which can be enumerated among the "anecdotes" as a literary genre since it is especially suitable to embody the logical contradiction necessary for any logical or other paradox by the opposition of common sense's implicit interpretation of the described situation versus the explicit meaning, which the paradox reveals suddenly following its logical chain.

Fortunately, Lewis Carrol is both writer and logician, so both of his paradoxes are simultaneously interesting short stories therefore containing connotations transcending corresponding formal structures being able to be represented exhaustively even only symbolically. They can be interpreted many times in different ways just as any literary text, after which new specific connotations are complemented by each interpreter being explained directly. One can debate whether one or another connotation are really available in the interpreted piece of literature, or it is rather created by the interpreter's imagination. Nowadays, that discussion is rare for any interpretation even seeming generated by a "sick mind" is equally admissible as well.

The specific connotation added to Carroll's two paradoxes able to unify them in virtue of his suggestable intention is the opposition of the traditional propositional logic of syllogisms versus the contemporary interpretation of propositional logic as Boolean algebra. Indeed, it seems admissible at the end of the 19th century when Carroll suggested them since the interpretation by Boolean algebra had appeared at that time generating the collision embedded as a hidden connotation eventually revealed by the present paper,

Furthermore, that connotation is in turn interpreted by the coined term of "logical unitarity" to be linked to the inherent unitarity of the separable complex Hilbert space, and by it to that of quantum mechanics versus the non-unitarity of the theory of quantum information and quantum computer, on the one hand, and to "Hilbert arithmetic" via the qubit Hilbert space, on the other hand, following the direction, objective and intention already of the present paper.

The genre of logical paradox situated on the cross of logic and literature inherently interprets the logical contradiction at issue as the sudden and unexpected end of an anecdote or a short story, if one considers it as a piece of literature rather than as a "dressed in words" formal structure. Thus, it calls for (as if) loose interpretation usual for literature, hermeneutics or philosophy, but not for the mainstream of logic.

Meaning all those conventions very relevant to Carroll's short stories designed as an ornament of a corresponding logical contradiction representable thoroughly formally, one can question about which are those purely formal nuclea of his paradoxes:

"A logical paradox" exemplifies the pair of syllogisms (as Carroll himself has written in his text): 
1. $(A, \neg B) \rightarrow C$

2. $A \rightarrow B$

$\mathrm{A}, \mathrm{B}$, and $\mathrm{C}$ are arbitrary propositions; The problem is whether $\mathrm{C}$ is restricted in any way by both conditions, and the answer is not, in virtue that a false premise of an implication does not restrict any conclusion, which can be both false or true due to other and non-formulated premises out of the scope of Carroll's "logical paradox".

Still one reflection is possible if the comma as usual is interpreted as the operation "conjunction" in Boolean algebra. Then, $(A, \neg B) \Leftrightarrow \neg(B \rightarrow A)$; so, $(A, \neg B)$ only excludes for " $A \rightarrow B$ " to be the particular case of logical equivalence (i.e. it implies " $\neg(A \leftrightarrow B)$ " as to " $A \rightarrow B$ "). Unifying both premises under that consideration, the result is: " $(A \rightarrow B) \rightarrow C$ ", or just " $A \rightarrow B \rightarrow C$ " in virtue of the associativity of implication. Thus, the opposite solution of the problem is inferred: so Carroll should mean just that a pair of opposite solutions of the same logical problem are valid, which justified the used word "paradox".

The interpretation in the present paper bounds the former solution with propositional logic as Boolean algebra, and the latter one, with the traditional logic of syllogisms (though represented by the contemporary symbols borrowed from Boolean algebra).

In fact, the two opposite solutions are due to the ambiguity of the enumeration of two different logical conditions. Indeed, the former interpretation means: " $[(A, \neg B) \rightarrow C] \wedge$ $(A \rightarrow B)$ ", while the latter interpretation: " $[(A, \neg B) \wedge(A \rightarrow B)] \rightarrow C$ " eventually justifying itself by the ostensible fact that only the composed propositions of " $A$ " and "B" are relevant to each other and thus " $\mathrm{C}$ " has to be out of the brackets. However, the same false substitution presupposes the conclusion that " $C$ " unambiguously depends on " $A$ " and " $B$ " (and in the final analysis, only on " $A$ " since " $B$ " depends on " $A$ " as well). In other words, the wrong substitution means the conclusion implicitly as a premise: an obvious logical fallacy.

Additional connotations to that formal structure are complemented by the exemplification of "A", "B", and "C" as spatial relations: "in - out" therefore meaning the implicit premise that "in" is the idempotent logical negation of "out": a statement valid in classical mechanics, but not in quantum mechanics. Thus, the connotation of "entanglement" can be added referring to the utilized exemplification of logical structure.

On the contrary (now meaning "What the Tortoise said to Achilles)", Boolean algebra naturally admits operations of an infinite set of operands, furthermore suggesting necessarily an unambiguous ultimate result of their accomplishment (though being an infinite set) ${ }^{9}$. The formal structure is:

1. $(A \rightarrow C) \rightarrow\{[A \wedge(A \rightarrow C)] \rightarrow C)\}$

2. "1" (just above) describes an iterative logical procedure, which can be repeated therefore ad lib or ad infinitum.

The same formal structure is interpreted in the present paper as violating of "logical unitarity" between the traditional propositional logic of syllogisms (as pre-supposed to be a

9 Probably, Lewis Carroll had noticed the analogy to Zeno's "Achilles and the Tortoise", which had perhaps inspired "What the Tortoise said to Achilles". 
thought process running in time) and the calculations in Boolean algebra (not linked to time, by itself, and thus allowing for calculations containing an infinite set of operands) ${ }^{10}$.

"What the Tortoise said to Achilles" seems not to contain any logical fallacy as a formal structure. Anyway, it involves implicitly infinity due to an iterative repetition ad lib and thus, ad infinitum. Indeed, the traditional propositional logic of syllogisms does not consider that kind of infinite syllogisms for they seem to be meaningless if one has interpreted a syllogism as a human thought running in time (that infinite syllogism would require an infinite time to end: a condition which is obviously meaningless). The foundation and justification is to bound unitarity in quantum mechanics and "logical unitarity" as the relation of a well-ordering to a "coherent state" (i.e. any well-ordering and thus, all well-orderings) in both cases.

\section{BOTH CONNOTATIONS OF THE PARADOX ("What the Tortoise ...")}

The formal logical structure does not exhaust at all the meaning, especially philosophically meaning of "What Tortoise said to Achilles"11. A few connotations are links to other areas, which situate the paradox among human cognition and can be qualified as its Fregean "Sinn" (i.e. "sense" versus "meaning": "Bedeutung") $)^{12}$. Those are a few exemplifications of the formal structure, namely:

1. "A" is a composite proposition, consisting of the conjunction of two propositions, which can be notated as " $\mathrm{A}_{1}$ " and " $\mathrm{A}_{2}$ ": " $A=A_{1} \wedge A_{2}$ ". Both " $\mathrm{A}_{1}$ " and " $\mathrm{A}_{2}$ " refer to a relation. That relation is the relation of equivalence (equality). " $\mathrm{A}_{1}$ " refers to two sides of a triangle; " $\mathrm{A}_{2}$ " refers to a general property of "equivalence". All those exemplifications are unified as a single one because they mean each stage of the paradox internally or separately unlike the second exemplification, which hints an analogue as a whole to the ancient aporia of Zeno:

2. That allusion is introduced borrowing both personages of Zeno's paradox: Achilles and the Tortoise, therefore suggesting a hidden formal structure able to underlie the ancient apporia and

${ }^{10}$ According to Brown (1954: 170), the "first moral" of "What the Tortoise said to Achilles" is: "the legitimacy of inferring that since $p, q$, does not require the truth of the statement that if $p$, then $q$ in the same way as it requires the truth of the statement that p". The same suggested difference is interpreted (in the present paper) as that between logic as syllogistic, on the one hand, and as Boolean algebra, on the other hand.

${ }^{11}$ Just many connotations rather than the proper logical structure are offered, for example, in: Moctefi and. Abeles, eds. (2016). One can also notice Ree's paper (1951) following Carroll's text literally to represent the author's logical ideas to it.

${ }_{12}$ Laraudogoitia (2014) suggests still one, but rather "hidden sense" of "What the Tortoise said to Achilles" by a continuation of the text, which can be granted to be a "logical extrapolation" of it demonstrating that "there are free actions (in general, contingent states of affairs) that can be predicted by means of purely logical reasons" (p. 405). Hilbert arithmetic by its inherent link to the formalism of quantum mechanics can elucidate the same idea additionally, but quite independently. For example, Conway and Kochen $(2006 ; 2009)$ "free will theorems" being directly inferable from that formalism under the condition of Lorentz invariance demonstrate the "free actions" e.g. of the investigated electron as predictable by the description of quantum electrodynamics. However, Hilbert arithmetic allows for even more: Boolean algebra (and thus propositional logic with its strict predictions) is to relate to the metalevel of free will (e.g. that of the electron at issue and described by a corresponding wave function and belonging to both qubit Hilbert space and Hilbert arithmetic, in a wider sense). 
Carroll's contradiction. Achilles cannot overtake the Tortoise just as an elementary implication cannot be accomplished being analogically intermediated again and again ad infinitum. The meant contradiction refers to "common sense" (as in his previous "logical paradox") stating the obvious: as Achilles will overtake the Tortoise very soon really, so the implication can be ended practically instantly.

Both paradoxes (Zeno's and Carroll's) do not contain any logical or mathematical fallacy, both involve infinity implicitly by a process repeatable iteratively ad infinium, therefore suggesting that our understanding of infinity contains some ambiguity or contradiction able to justify the conclusions in both cases, however contradicting empirical experience.

Furthermore, the unification of the two paradoxes (the one being physical or mechanical, the other one, logical) suggests the Hegelian idea about the philosophical category of "motion" able to unify the two sides of the Cartesian dichotomy of "mind" (or particularly, logic meant by Carrol's paradox) and "body" (mechanics or physics meant by Zeno's paradox). In fact, the category of motion can represent ontologically Kant's transcendental solution (being traditionally related rather to epistemology, to "mind", though it implies by itself to be not less relatable to "body") of Descartes's problem (eventually the fundamental one of the modern Western philosophy).

Thus, Helel exemplified "motion" historically, to emphasize his ontological interpretation of Kant's transcendentalism: both to history of philosophy and to philosophy of history. Even more, he introduced "dialectic logic", which being ontological is what should correspond to "formal logic" (i.e. only to the traditional logic of syllogisms since in Hegel's age, the interpretation of propositional logic as Boolean algebra had not appeared yet). One can add that Heggel's only and properly philosophical approach influenced crucially the course of history in the 20th century by means of Marx's reinterpretation as "dialectical and historical materialism" (and embodied as the "scientific doctrine" of the "real socialism" in the Soviet Union and all other countries more or less controlled or impacted by it).

The present section of the paper will restrict itself only to still one exemplification of the possible unification of logical and physical (mechanical) paradoxes, namely those of the "Liar"13 and of the "Arrow", thus directly borrowing Carroll's kind of allusion:

One can suggest that both paradoxes being both ancient and famous need not be represented. Even more, the pathway of their analogy seems to be obvious, or speaking loosely or metaphorically: the "Arrow" "lies" that "it is here"; the "Liar" moves his or her statement (about what she or he has said) not to be identifiable with itself therefore being in a kind of "logical motion"14.

\footnotetext{
${ }^{13}$ The paper of Aerts, Broekaert, and Smets (1999) interprets the "Liar" by the mathematical formalism of quantum mechanics, the separable complex Hilbert space. Thus, it anticipates the radical and fundamental approach of the present paper stating in the final analysis that Hilbert arithmetic (respectively, the qubit Hilbert space) is able to resolve the real paradoxes of logic (along with those of mathematics or physics).

${ }^{14}$ That analogy moreover suggesting an underlying shared formal, logical and mathematical structure of both paradoxes is discussed in detail in: Penchev 2009.
} 
That observation can be further represented in detail and logically consistently by the following formal structure: if " $A$ " is false, " $A$ " implies the logical negation of " $A$ ", So, let " $A$ " be "The arrow is here": indeed, that "The arrow is here" is false, implies that "The arrow is not here". Analogically, let "A" be "The liar is lying" (therefore emphasizing that he or she lies just saying "I lie"): then if "The liar is lying" is false, this implies that "The liar is not lying". Thus, there exists no formal logical contradiction in both cases for " $\mathrm{A}$ " can be true or false in both cases though the paradoxes mean the option of "false". The alleged contradiction is due to the confusion of " $A \rightarrow \neg A$ " (which can be as true, where " $\mathrm{A}$ " is false, as false, where " $\mathrm{A}$ is true") with " $A \wedge \neg A$ " (which is identically false).

One can notice that the formal structure implies that both paradoxes are also valid if as " $\neg A$ " is granted as " $\mathrm{A}$ " in the notations above. That idempotent pair is sometimes interpreted as the definition of the Hegelian "dialectic contradiction" or the Marxist "objective contradiction" (e.g. Petrov 1971), namely and formally:

$$
\text { "Contradiction }(\text { def }) \equiv[(A \rightarrow \neg A) \wedge(\neg A \rightarrow A)] \Leftrightarrow(A \leftrightarrow \neg A) "
$$

One can easily watch that both "Liar" and "Arrow" do not satisfy that definition for the idempotent exchange of " $A$ " and " $\neg A$ " in them means the logical operation of "disjunction", but quite not, "conjunction". Thus, the alleged "definition of objective contradiction" turns out to be an identically true tautology:

$$
\text { “Alleged Contradiction (def) } \equiv[(A \rightarrow \neg A) \vee(\neg A \rightarrow A)] \Leftrightarrow \neg(A \leftrightarrow \neg A) \text { " }
$$

Thus, the exemplified meta-allusion of "What the Tortoise said to Achilles" by the pair of the "Liar" and "Arrow", furthermore containing still one very essential allusion to Hegel's doctrine of motion as ontological, is able to suggest the following conjecture referring to both pairs: (1) Zeno and Carroll's paradoxes; (2) the "Arrow" and the "Liar":

The conception of infinity can be "repaired" (meaning Gödel's dichotomy about the relation of (Peano) arithmetic to (ZFC) set theory: either incomplete or contradictory) investigating the condition under which the contradiction of finiteness and infinity can be transformed into an "alleged contradiction" (as it is defined just above rigorously). Particularly and very important as to Gödel's dichotomy, this means a dual counterpart of Peano arithmetic to be introduced so that if " $\mathrm{A}$ " is to mean: "Peano arithmetic is available", " $\neg A$ " should be able to mean "The dual counterpart of Peano arithmetic is available" (i.e. being able to be equivalent to "Peano arithmetic is not available").

Meanwhile, one has to notice that Bohr's quantum complementarity (initially applied to, or extracted from quantum mechanics; then, generalized to many sciences and to philosophy) satisfies just the transformation of "dialectic contradiction" into an alleged contradiction relatively painlessly: or in other words, that of Hegel's ontological motion into quantum motion (however very often restricted only to area of quantum physics or sciences utilizing its results).

Then, the conjecture which means that Bohr's quantum complementarity can be considered as a universal solution of all paradoxes whether ancient or contemporary, whether logical, 
mathematical or physical, seems to be reasonable. Furthermore, the structure of Hilbert arithmetic can embody that universal principle of quantum complementarity ...

IV THE UNIFIED PROBLEM OF CONCLUSION, MOTION AND EQUALITY IN A HELELIAN MANNER

Hegel's doctrine, though originating from Kant's transcendentalism, possesses a fundamental specific feature: it means a unification of logic, philosophy, history and even physics by a kind of "philosophical logic" called dialectic logic. However, that "dialectic logic" turned out to contradict formal logic rejecting the law of (non-)contradicting therefore postulating for itself to be a constitutionally non-falsifiable (or "metaphysical" after Popper) dogma without any scientific value, allowing for an arbitrary statement to be inferred and thus "proved" by it in the final analysis.

Many, many attempts have been undertaken to "repair" propositional logic modifying its axiomatic basis in a way to make dialectic logic both formal and falsifiable ${ }^{15}$. None of them has received recognition and distribution outside the specialized field of non-classical logic.

The idea of Hilbert arithmetic shares the same intention, but approaches radically differently: it does not repair propositional logic in any way, i.e. conserving it thoroughly and literally. What is "mended" is dialectic logic utilizing the "lesson" of nature, thought out by quantum mechanics and reflected philosophically by Niels Bohr in his principle of complementarity interpreted formally and logically. This is described above by the substitution of the definition of "dialectic (or objective) contradiction" by that of "alleged contradiction", furthermore interpreted to be isomorphic to complementarity.

Then, the relation of logic, arithmetic, and set theory is to be modified in a way to be complementary (in that sense of "alleged contradiction") to the usual separable complex Hilbert space therefore realizing the goal of Hegel's dialectic logic, however inherently differently and consistently to propositional logic: namely, the unification of logic, philosophy, and physics in their, thus shared foundations.

This can be demonstrated particularly to Hegel's category of "motion" being ontological, i.e. underlying all of those: mechanical motion, physical or mental change, logical conclusion, historical change in both history and history of philosophy. They can be merged as different aspects of Hilbert arithmetic, sufficient for that objective. Accordingly, mechanical motion are

\footnotetext{
${ }^{15}$ Along with the huge set of paraconsistent logics, there exist many quantum logics, which in turn modify some axioms of propositional logic. The conclusions of quantum mechanics have seemed so extraordinary that one can admit that it needs a new, "quantum" thinking and thus, a new, "quantum" logic whether generalizing or modifying (in a narrow sense) traditional one. The present paper does not share that motivation incited to creating new logics, and its relation to non-classical logic including all quantum logics is elucidated in detail in Section X: there is no need of new logics, but one need clear up and resolve the problem of finiteness and infinity (shared by the foundations of mathematics and thus, by mathematics at all) as to traditional logic and Boolean algebra also in their concepts and terms. The reason is that all non-classical logics can be situated on the side of classical logic in virtue of their models in it versus its interpretation as a mathematical structure (such as Boolean algebra) and embodied as a problem in the formal structure of "What the Tortoise said to Achilles" chosen to be the topic of the paper just for that.
} 
Hermitian operators as well as any physical change; mental change can be represented analogically (and directly interpreting the foundation of quantum mechanics: Penchev $2021 \mathrm{July}$ 26). Logical conclusion can be repeated isomorphically to the standard definition in the framework of Hilbert arithmetic. The mediation of Husserl's phenomenology interpreted absolutely formally resolves the problem of how the historical change in both history and history of philosophy can be embedded in the same framework (Penchev 2020 December 14)). So, one can state that Hilbert arithmetic is able to embody the Hegelian (including Marxist) philosophical and scientific ideas exhaustively, however absolutely consistently to propositional logic, and thus, to science (including experimental science).

Meaning the consideration just above, Lewis Carroll's "What the Tortoise said to Achilles" can be interpreted as kind of "foreshock"16 anticipating Hilbert arithmetic, especially by its connotations of merging mechanical motion (meant by Zeno's paradox literally) and logical conclusion (suggested by Carroll), already explained from the present viewpoint, on the one hand, and logical equivalence and quantitative equality, on the other hand, therefore needing a notice, which follows;

The category of identity is central in Hegel's doctrine (opposing "philosophical identity" including "change" versus the traditional formal and logical identity excluding any change). It serves to justify "dialectic logic" and simultaneously (formally and logically, in a proper "vicious circle") philosophical identity to be inferred from dialectic logic.

Dialectic identity which means simultaneously "change" has been shared by many left radical parties and movements (as their "ideologies" or political philosophies) preaching "revolution" meaning an extremely different, new political power and social order often getting the idea of violence for it to be able to happen. The paper, however, discusses "dialectic identity" in an abstract, logical and properly philosophical aspect, thus absolutely independent of the above connotations, which are social and political: i.e. they would be irrelevant to the present context.

In fact, the use of "identity" includes "change" to some extent, fussily determined, due to which it is a subject of a well-known logical paradox: the "sorit paradox". Quantum mechanics also suggest a solution of it since it was forced to resolve an analogical scientific puzzle: how to unify consistently the description of both: (1) the leaplike, quantum change of the studied microscopic entity; and (2) the continuous and even smooth change of the macroscopic apparatus obeying the differential equations of classical mechanics:

Its solution suggests a wave function able to describe that unification, and thus. a corresponding probability distribution, to which the wave function at issue is its characteristic function: that distribution can be interpreted as a solution of the sorit paradox therefore presupposing that "identity" is transformed into "change" both gradually and probabilistically,

\footnotetext{
${ }^{16}$ Philosophy of science follows the natural attitude of human experience and science therefore describing history of science as a causal process over time and thus excluding any reverse causality by the influence of any future events on any present phenomena. However, if one has introduced a quantum worldview to be leading as here, the corresponding philosophy of science should discuss "foreshocks" (along with the standard causal "aftershocks") in history of science or history at all.
} 
after the sorit paradox has been extrapolated in advance into the terms we are properly interested in: those of the Hegelian or marxsist "dialectic change":

Following concepts of the dialectic law of how "quantitative changes pass into a qualitative change", one can distinguish the changes within the same identity as quantitative versus the change meant in a proper sense: i.e. generating a new quality. Then, the corresponding solution of quantum mechanics would state that both quantitative changes and qualitative change(s) share the same probability distribution (implying a wave function as its characteristic function unambiguously), so that it is a mapping of the set of quantitative changes into the probabilities of the qualitative change(s) to be observed empirically or experimentally. In other words, if that probability distribution is visualized as a two-dimensional graphic, its abscissa would correspond to the quantitative changes conserving the same identity, and its ordinate accordingly, to the probabilities of qualitative change(s) implying a new identity.

Meaning the concepts of Hilbert arithmetic introduced rigorously and in detail in other papers (e.g. Penchev 2021 August 24)), that mapping means the probability distribution (or a wave function as its corresponding characteristic function) of finite quantitative (literally, arithmetic) changes to be considered as the new quality of infinity. In other words, Hilbert arithmetic can be considered as a relevant tool to describe "dialectic identity" (respectively, "dialectic change") rigorously and consistently to propositional logic.

One meets still one trouble, rather properly logical and mathematical than philosophical, discussing identity and referring to the problem whether logical equivalence (notated further as $" \Leftrightarrow ")$ can be treated to be the same as quantitative (mathematical) equality (further, "="); symbolically and particularly as to the transitivity of both sharing the same formal structure of a "relation of equivalence", where they can be interpreted correspondingly as logical equivalence and mathematical equality:

Does the proposition " $a=b=c$ " mean the same as the proposition " $a \Leftrightarrow b \Leftrightarrow c$ "? If "Yes", are " $=", " \Leftrightarrow "$ mutually interchangeable? For example, does " $a=b \Leftrightarrow c$ " make sense? Properly, the question relates to the self-referential use of both logical equivalence and mathematical equality as two exemplifications of the relation of equivalence. Indeed, one can ask analogically: if " $a=b$ " (respectively, " $a \Leftrightarrow b$ "), do " $a$ " and " $b$ " mean the same?

The problem can be generalized, e.g. so: can one exchange arbitrarily the interpretations in a framework of the same class of equivalence? Its basis is the puzzle about the admissibility of self-predicativity: whether or where a level and the corresponding next metalevel can be mixed without any logical fallacy.

One can distinguish the indefinitely (infinitely) increasing hierarchy of levels, each of which can be enumerated by a natural number, on the one hand, and an idempotent structure consisting of only two levels so that each of them can be considered as the metalevel to the other one and thus being mutually notatable by "logical negation", on the other hand. The latter case is realized in any mathematical structure able to be dual, such as the separable complex Hilbert space of quantum mechanics and borrowed in Hilbert arithmetic. 
At the same time, it is able to embody the indefinite (infinite) hierarchy always notatable by natural numbers, however as if ordering it to be situated on the sublevel to that of idempotency. In fact, this is only seeming, and can be easily fitted by introducing the qubit Hilbert space (being equivalent to the separable complex Hilbert space under only technical conditions) as it: as a whole is contained in any qubit; thus, restoring the perfect symmetry of hierarchy and idempotency for which it is inherently designated.

Meaning the above observation, one can ignore the distinction of the initial sets (which are different arguments of the mappings), of both cases of equivalence and equality: propositions in the former case, but elements of any set in the latter. However, the much more important statement is that Hilbert arithmetic is a relevant tool to be unified (and thus, to be ignored the corresponding distinction) of the logical mapping into only two elements (e.g.: "false" and "true") and assignable to idempotency, and the mathematical mapping in an infinite set (e.g that of all natural numbers), i.e. assignable to hierarchy.

If one takes in account, that the set of two elements is able to represent any finite set (e.g. as the Turing machine tape is able to demonstrate), the unification of idempotency and hierarchy, accomplishable as Hilbert arithmetic by the qubit Hilbert space, resolves simultaneously the fundamental mathematical and philosophical problem how to be unified finiteness and infinity (e.g. exemplified by the Gödel dichotomy about the relation of (Peano) arithmetic to (ZFC) set theory).

One is to notice furthermore that the idea of idempotency and hierarchy to be unified (along with its embodiment in Hilbert arithmetic) is embedded still in the axiom of choice (standardly enumerated among the axioms of set theory though it can be interpreted as a meta-axiom regulating the relation of arithmetic and set theory), and more precisely, in the equivalency of the axiom of choice (for idempotency) and the well-ordering "theorem" (for hierarchy).

On the ground of those preliminary notices above, one can conjecture that the connotation (conventionally enumerated as "second") meant by "What the Tortoise said to Achilles" and referring to "equality" hints at the eventual unification of idempotency and hierarchy, underlying Hilbert arithmetic.

V THE MAIN PROBLEM OF QUANTUM MECHANICS AND THE "PROBLEM OF THE TORTOISE AND ACHILLES" AS THE SAME

Meaning the above, logical and physical discussion of Zeno's and Carroll's paradoxes in a "Hegelian manner", one can speak of a single paradoxical structure underlying both and rather referring to the relation of finiteness and infinity, and more precisely to common sense's belief that, speaking loosely, infinity is always "much more" than finiteness and thus, postponing ad infinitum the moment when Achilles (on the side of finiteness) overtakes the Tortoise (on the side of infinity) whether physically (after Zeno) or logically (after Carroll).

One can use the metaphor (or structure) of the Tortoise and Achilles to quantum measurement, adding still one paradoxical aspect of the problem: for example as follows. The moment of overtaking can be interpreted to correspond to the equality of a discrete quantum 
measure (naturally meant by the Planck constant) and a continuous classical measure (embedded in the apparatus since it is described by classical mechanics $)^{17}$.

Then the additional antinomic aspect would consist in the necessary interpretation of Achilles to be "quantum" (so, rather microscopic) versus the macroscopic "Tortoise of the apparatus", which is to be classical. One can describe that new, exchanged "quantum" reinterpretation so: the huge, being macroscopic, Tortoise can never overtake the microscopic quantum Achilles though any of his leaps must correspond exactly to the extremely small Planck constant. The reason is that the Tortoise's step has to be infinitely small since she or he moves continuously as classical mechanics requires necessarily, and any finite quantity (such as the Planck constant in the case) is much greater (even, infinitely greater) than the Tortoise's step, which is to be infinitely small for his or her motion to be continuous. So, the same prejudice of common sense about the relation of infinity and finiteness takes place, though modified to the relation of an infinitely small quantity and an extremely small, but finite constant.

As it is explained above, Hilbert arithmetic rejects common sense's "infinity is always greater than finiteness" (respectively, any finite quantity to be always greater than an infinitely small one ${ }^{18}$ ) involving both in the same, but dual structure, furthemore isomorphic to the qubit Hilbert space (and thus, to the usual separable complex Hilbert space of quantum mechanics under relevant conventions).

So, the justification of the above "ridiculous", "quantum" reinterpretation of the paradox about quantum measurement intentionally consists in the relativity of finitiness and infinity in a "Skolemian manner", but established a long time ago also in the Standard model as the relativity (respectively, identity) of the local space (e.g. the quantum Achilles's one) and the global space (e.g., that of the macroscopic, "classical" Tortoise). The sense of that reinterpretation is, speaking loosely, in the logical equivalence that if Achilles cannot overtake the Tortoise, the Tortoise cannot also overtake Achilles as well as vice versa.

The usual understanding of common sense about quantum mechanics restricts its subject only to microscopic, quantum phenomena commensurable with the magnitude of the Planck constant: thus, it is relevant to macroscopic phenomena very rarely, under too special conditions, but never directly. On the contrary, other papers (e.g. Penchev 2020 July 16) explain the main problem of

\footnotetext{
${ }^{17}$ For example, Rees (2001) (as well as many other authors) describes quantum phenomena in terms of Zeno's paradoxes.

${ }^{18}$ One should mention the "Archimedes axiom" and the substitution of it by its negation in Abraham Robinson's (1966) "Nonstandard analysis", even more so related to the axiom of choice by the weaker version of the latter: the "lemma of ultrafilters". The negation of Archimedes's axiom supplies finiteness and infinity to be distinguished from each other again, however in a different way in comparison with the "standard analysis": both finite small constant and (Leibniz) "differential" of nonstandard analysis can be actual and thys similar to each other, but just the negation of Archimedes's axiom allows for them not to be compared. On the contrary, standard analysis divides them into different qualities (correspondingly, either "finite" or "infinitesimal") conserving to be comparable in virtue of Archimedes's axiom, but only by infinite quantities. So, both standard and nonstandard analyses share the same dichotomy being supported by the corresponding alternative within the same dichotomy about finiteness and infinity: either comparable different qualities or the same quality in two incomparable ("complementary") aspects. The latter alternative seems to be shared by both quantum mechanics and nonstandard analysis.
} 
quantum mechanics to be: how the discrete, quantum entities to be consistently described by the continuous measure of the apparatus according to classical mechanics, or in other words, how to be described uniformly both discrete and continuous phenomena. Then, any entity to be quantum is not more an absolute property (as the former viewpoint of common sense presupposes), but a relation; thus, if its premises be satisfied, macroscopically observed phenomena (e.g. those of entanglement) can obey quantum correlations directly.

The present paper continues further, extrapolating the relative interpretation of quantum mechanics (after which "to be quantum" is not more a property, but a relation) to the problem of finiteness and infinity following its exemplification by both versions of the paradox about Achilles and the Tortoise. Just as Scolem (1922) has suggested following the conclusion of set theory, and more especially, the axiom of choice, that finiteness and infinity should be considered to be considered rather as relations than as properties of sets.

The justification of linking the relative interpretation of quantum mechanics (in the sense as above) and Scolem's "relativity of "set" consists in the axiom of choice postulated in set theory and inferrable in quantum mechanics ${ }^{19}$ (then embedded in the unitarity of Hilbert space). Then, the relativity of finiteness and infinity can be realized to resolve as Zeno's physical paradox as Carroll's logical paradox therefore bridging both to the main problem of quantum mechanics if it is understand so: how to be reconciled, unified, and identified (in the final analyses) as the same both the discrete quantum description of the investigated entity by itself and its continuous readings by the apparatus in turn necessarily represented only by the smooth differential equations of classical mechanics.

\section{THE SOLUTION OF QUANTUM MECHANICS AS A SOLUTION OF THE CITED ANTINOMIES}

If the main problem is established to be just as it is formulated at the end of previous paragraph, its solution consists in the introduction of the unitarity embedded in the separable complex Hilbert space as a necessary condition to be unified the discrete viewpoint of Heisenberg's matrix mechanics (conventionally attachable to the measured or investigated microscopic quantum entity "by itself') and smooth one of Schrödinger's ondulatory (realizable as what the apparatus registers to that entity). In fact, the Schrödinger equation elucidates only the way for both descriptions to be universally equated if the separable complex Hilbert space as containing any possible solution ("wave function" as its element) is granted in advance.

Nowadays, the theory of quantum information interprets it as the qubit Hilbert space therefore underlying the quantum generalization of information (equivalent to the information of infinite sets or series: Penchev 2020 July 10). Thus, quantum information can be considered not only as the universal substance of the physical world (e.g. Penchev 2020 July 24), but also and not less, as unifying what any empirical or experimental experience referring to physical body means with what its mathematical description by mind means (е.г Penchev 2021 February 26) just in virtue of the completeness of quantum mechanics verified by the theoremes of the absence

\footnotetext{
${ }^{19}$ That idea is developed in detail in other papers (e.g. Penchev 2021 April 12).
} 
of hidden variables (Neumann 1932; Kochen, Specker 1967) as conclusion inferable only from the properties of the separable complex Hilbert space (respectively, the qubit Hilbert space).

So the completeness of quantum mechanics can be reinterpreted as originating directly from the use of a special mathematical structure rather than as implied by quantum mechanics as a whole. As far as it is a mathematical structure (thus not needing any physical premises), it can be involved for resolving the "annoying" incompleteness of the foundations of mathematics (after the Gödel dichotomy about the relation of arithmetic to set theory as first-order theories to propositional logic).

However, all history and successes of mathematics suggest indirectly that mathematics should be grounded very well de facto. Nonetheless, Gödel's direct reflection on the foundations doubts the intuitive confidence of almost all scientists rather than only mathematicians. One can admit that there is a problem in the reflection on its foundations rather than that in itself as a huge cognitive building since and for millennia.

I have already argued in other paper (Penchev 2020 August 25) that the Gödel dichotomy about the relation of arithmetic and set theory can be related as a statement to itself (i.e. self-referentially) thus transforming into an independent additional axiom rather than a proper theorem directly inferable from the explicit premises of arithmetic, set theory, and propositional $\operatorname{logic}^{20}$. Its dichotomy can be traced back to the relation of the axiom of induction in arithmetic (implying that all natural numbers are finite) and the axiom of infinity in set theory (postulating that the set of all natural numbers is infinite).

Then, the use of both axioms into a single proof (as the Gödel paper published in 1931 does) continues an ambiguity needing implicitly an additional axiom about how to interpret their relation if both are simultaneously used as the formulations of theorems in the paper force. Properly, that non-formulated additional axiom is equivalent to the central dichotomic conclusion of the paper about the relation of arithmetic (containing the axiom of induction) and set theory (containing the axiom of infinity); namely: either incompleteness or inconsistency.

In fact, that conclusion is a direct corollary from the analogical relation presupposed to regulate the ambiguity of both axioms implicitly meaning that all natural numbers are correspondingly either incomplete or inconsistent to the set of all natural numbers.It means informally that "set" is a substantive and independent mathematical entity thus irreducible to its elements and indirectly justifying set theory as one of the "whales" supporting mathematics.

Its basis can be revealed in the organization of contemporary cognition rather than in mathematics itself: our "episteme" (by Michel Foucault's term and concept) originates from Cartesian dualism opposing "mind" to "body" and then creating a model of that opposition within mathematics itself by arithmetic itself (for "mind") and set theory (for "body") therefore in turn being dichotomic or contradictory. Indeed, the availability of such a model in mathematics suggests for it to contain the world as a whole (just as it contains the model at issue), on the one hand, but on the other hand, mathematics is not less contained within the world just as "mind" (for mathematics) in "body" (for the world).

${ }^{20}$ The idea is represented for the first time in: Penchev 2010 (in Bulgarian). 
Then, the Gödel incompleteness axiom (i.e. not a "theorem" after the present consideration) chooses only the latter aspect (mathematics within the world) ignoring absolutely the former one (the model of mathematics and the world within mathematics itself). So, that Gödel incompleteness axiom is rather a meta-axiom postulating how to be interpreted the relation of mathematics and the world: namely, situating the latter within the former (which seems to be natural for empirical experience, experimental science and the contemporary organization of cognition dominated by them).

This fundamental branch of mathematics accepting or implicitly granting that meta-axiom can be called Gödel mathematics. On the contrary, if mathematics shares the negation of the Gödel incompleteness axiom (namely, the former aspect of the world within mathematics in virtue of the availability, neglected by Gödel, of the relation of mathematics and the world within the former), it can be called Hilbert mathematics.

All subareas of mathematics not needing the simultaneous use of arithmetic and set theory (more precisely, the simultaneous use of the axiom of induction and axiom of infinity) can remain the same in both Gödel and Hilbert mathematics and they comprise almost all mathematics. Nonetheless, there exists a few fields in which the dichotomic choice between them is unavoidable, and the foundations of mathematics (as the Gödel incompleteness demonstrates itself) or quantum mechanics (in virtue of the theorems of the absence of hidden variables implies) are among them.

Indeed, one can trace back the pathway in which Hilbert mathematics naturally underlain by Hilbert arithmetic supplies an alternative relation of the axiom of induction and the axiom of infinity and then and thus, a different solution of the Gödel problem about the relation of arithmetic and set theory in the foundations of mathematics.

It is grounded on the interpretation of the relation of arithmetic and set theory (respectively, that of the axioms of induction and infinity) also by means of the idea of "alleged contradiction" implying for them to be complementary to each other: or in other words, both arithmetic and set theory are applicable, but never simultaneously. So, the contradiction between them is never actual, and only potential since one has to choose whether the axiom of induction (thus in the framework of arithmetic) or the axiom of infinity (and thus, in the framework of set theory) to use in each case $^{21}$.

Hilbert arithmetic can be considered as a structure inferred from the qubit Hilbert space (in turn originating from the separable complex Hilbert space of quantum mechanics) and designated just for supplying the complementary use of "either arithmetic or set theory", furthermore each of them to be complementary to the utilization of the qubit Hilbert space thus interpreting any entity and issue as physical rather than as mathematical:

Obviously, that formal solution based on Hilbert arithmetic can be generalized to the fundamental problem of Western philosophy originating from Cartesian dualism: "mind" and

\footnotetext{
${ }^{21}$ Speaking loosely, the sense of the axiom of choice can be interpreted as the necessary and dichotomous choice to be used either the axiom of induction (and then, the axiom of choice has the form of the well-ordering theorem) or the axiom of infinity (and thus, the proper axiom of choice).
} 
"body" to be complementary to each other and this to be the way for regulating of both kinds of description: either in terms of "body" (for the physical description by the qubit Hilbert space) or in terms of "mind" (for the proper mathematical description in terms of Hilbert arithmetic in a narrow sense).

Then, Zeno's paradox is to be interpreted in two complementary ways, after which the contradiction would appear only if one confuses both descriptions: on the one hand, either mathematical or physical, or on the other hand: either arithmetic or set-theoretical if the case is former, i.e. a mathematical description.

Indeed the physical description is what is naturally granted to be the real one in our "episteme", furthermore relevant to empirical human experience, and in which Achilles overtakes the Tortoise unambiguously and after a finite interval of time. The same is valid in the framework of one of both possible mathematical descriptions, namely that in terms of set theory. Nonetheless, the statement is false in the other one of them, in terms of arithmetic. However, the contradiction can be never actualized in virtue of the complementarity of both descriptions:

Speaking loosely, Achilles needs infinity weather mathematical or physical (realizable by the concept of continuity) in order to manage to overtake the Tortoise; thus, an imaginary Achilles competing with the Tortoise within an only arithmetic "reality" can never win, but our usual reality granted by human experience is not arithmetic: anyway, it can be consistently granted to be mathematical, but only set-theoretical though the completeness of mathematics needs its complementary counterpart, which is arithmetic.

The same kind solution can be immediately interpreted in the terms of Carroll's paradox in virtue of the isomorphism: the implication cannot be ever ended in a proper arithmetical reality ${ }^{22}$, but propositional logic is standardly related to physical reality and thus, to mathematical reality meant in set theory. Indeed, this is a result indirectly reconfirmed by the Gödel (1930) completeness paper. Thus, Hilbert arithmetic is able to describe "What the Tortoise said to Achilles" equally well both physically and logically.

VII THE PROBLEM OF THE COMPLETENESS OF MATHEMATICS BY THAT OF QUANTUM MECHANICS: THE IDEA OF HILBERT ARITHMETIC

An experimental science such as quantum mechanics is privileged in the episteme of Modernity in comparison with mathematics (originating from the "mind" in the same framework) to aspire to "completeness" as far as all mental models (among which the mathematical ones are) ought to obey empirical and experimental experience (collected and generalized e.g. by quantum mechanics as in the case). Independently of that "natural attitude" confessed by the scientific "mainstream" nowadays at least implicitly, the proof of completeness of quantum mechanics based on the theorems about the absence of hidden variables in it is properly mathematical: not needing or even admitting referring to experiments. Particularly, the link from them to entanglement is not logically necessary: its phenomena are only consistent

\footnotetext{
${ }^{22}$ How arithmetic can be interpreted as first-order logic if that is the case is discussed in detail in Section IX.
} 
with the absence of hidden variables and can be interpreted as "confirmations" only in that practical sense (unlike any eventual refutation).

So, the actual and thus acting episteme can be "surrounded" de facto, suggesting that the qubit Hilbert space being an only mathematical structure is sufficient to supply the completeness of mathematics (cherished more than a century) though expected initially for quantum mechanics in virtue of what the general organization of cognition in Modernity can accept or "swallow" (since the eventual completeness of mathematics implies an epistemic "revolution" and thus an earthquake in science at all).

Nonetheless, the proof of completeness though applied to quantum mechanics (obeying the dominating prejudice of common sense) is mathematical by itself and therefore, it is able to resolve the crisis in the foundations of mathematics revealed still in the beginning of the $20^{\text {th }}$ century. In other words, Hibert arithmetics only develops the completeness of the separable complex Hilbert space in the relevant mathematical concepts merely neglecting an eventual cognitive earthquake which it can cause (and many aftershocks in all sciences).

So, the troubles (rather than problems) about Hilbert arithmetic are philosophical, needing a new place of mathematics in cognition as well as the relevant rethinking of its relations with physics and philosophy after needing their unification and merging the three foundations. The corresponding shift of the worldview leads to a new, contemporary form of Pythagoreanism, which can be called "quantum neo-Pythagoreanism".

The essential corpus of basic mathematical ideas to underlie Hilbert arithmetic can be reduced to a few. The arithmetical units to be defined as classes of equivalence of qubits sharing the corresponding arithmetic unit at issue, i.e. "empty qubits" or those of a quantum Turing machine tape before recording any value in each qubit of it. Thus, it is natural to be complemented by the corresponding (Peano) arithmetic originating from the dual qubit Hilbert space therefore both satisfying Peano axioms, but avoiding the direct contradiction to the axiom of infinity in set theory (as in the Gödel incompleteness theorems) due to the set-theoretical complement of the dual Peano arithmetic to the set of all natural numbers. After that, propositional logic and set theory can be identified as isomorphic to each other as the same Boolean algebra and then both, as isomorphic to a nonstandard interpretation of Peano arithmetic (which, speaking more precisely, should be called "anti-nonstandard", or "anti-standard"23), in which the function successor is defined to be by adding a new member of equality rather than an arithmetical unit (e.g. Penchev 2021 August 24; or Penchev 2020 August 25).

At last, the dual counterpart of Peano arithmetic can be naturally interpreted as a qubit Hilbert space, therefore generating the representation of Hilbert arithmetic (in a narrow sense) and the qubit Hilbert space as dual to each other, and thus, the duality of mathematics and physics unifiable by a relevant Pythagorean kind of philosophy. The axiom of choice generates a

${ }^{23}$ The addition of "anti-" is due to the anti-isometry of the dual qubit Hilbert space (originating from that of the dual separable complex Hilbert space): to be "anti-nonstandard" (or respectively, "anti-standard") means the non-standardness being idempotent to be interpreted by anti-isometry also being idempotent. 
mapping of any infinite set in natural numbers, but it cannot be constant, but variable (even fundamentally randomly variable) for avoiding the contradiction of "all natural numbers" to the "set of all natural numbers": thus, the "backdoor" of the set-theoretical "Dedekind finiteness" (rejecting only the constant mapping of an infinite set into it finite subset) can be utilized and then mapped bijectively (only in virtue of the axiom of choice and the well-ordering "theorem") into the dual counterpart of Peano arithmetic, in which any unit can be already interpreted as a certain value "recorded" into the $n^{\text {th }}$ empty qubit after mapping.

Then, set theory being a first order logic of propositional logic (embedded in advance into the "anti-nonstandard" interpretation of Peano arithmetic) is also doubled due to the axiom of choice as: (1) Boolean algebra isomorphic to that of propositional logic (this is the one of both doubled copies of set theory, namely that before applying the axiom of choice); (2) two dual to each other, but standard Peano arithmetics and each of which satisfying the Gödel dichotomy and thus, either incomplete or inconsistent to set theory:

Particularly and as it will be discussed in detail in Section IX, "What the Tortoise said to Achilles" can be interpreted as a statement inverse to Gentzen's "cut elimination rule" (Gentzen 1935), but being false unlike the latter. In other words, even an infinite series of implications can be "cut", but no finite series of implications can imply an infinite one, and thus, the syllogistic scheme suggested by the Tortoise (the number of syllogisms of which is always finite in virtue of the axiom of induction) can never end. The paradox is "true" in that sense: i.e. this is a true statement (namely that the proposition reverse as above to the cut elimination rule is false), which contradicts only the prejudice of common sense.

\section{HILBERT ARITHMETIC AS THE “CLASS OF ALL CATEGORIES” (in category theory)}

Category theory is a proper mathematical theory referring directly to the foundations of mathematics. Unlike set theory (being historically first), category theory shares fundamental philosophical ideas about the unification of physics, mathematics, and even philosophy just in their common "root" in cognition and reality (e.g. Kuś and Skowron, eds. 2019; Krömer 2007; Giandomenico, ed. 2006; Peruzzi 2006, etc.) That is just as the worldview of the present paper though adhering to the more traditional approach meaning arithmetic, set theory and propositional logic as underlying mathematics, but relevantly modified as aspects of Hilbert arithmetic, in turn isomorphic to the formalism of quantum mechanics. Then, a natural conjecture is the similarity of Hilbert arithmetic and category theory can be discussed even as one or another degree of mathematical and philosophical equivalency:

An analogue of the basic ideas as they are sketched above can be discovered in category theory attempting to suggest an alternative viewpoint to the classical foundations of mathematics based on propositional logic, arithmetic, and set theory eventually overcoming the accompanying antinomies. The natural conjecture is that analogue can be formulated rigorously and mathematically: that is as an isomorphism in the final analysis.

Category theory intends to classify all mathematical structures in categories according to their axiomatic definitions and to describe the transformations between structures both within a 
category and from a category into another. Those transformations ("functors") are interpreted as mappings ("morphisms") but rather of logical collections of essential features and established by the corresponding axioms than structures (i.e. only extensionally representable as elements of sets).

Thus, the distinction of propositional logic and both fundamental first-order logics of arithmetic and set theory seems to be partly removed and partly substituted by the newly-invented distinction of categories (relevant rather to first-order logics) and functors or morphisms (describing their transformations rather logically).

The viewpoint to logic (including propositional) logic is also modified corresponding to the contemporary approach as a specific class of structures (e.g. the category of lattices) rather than the traditional one as series of syllogisms and relevant only to propositional logic (extremely restricted only to Boolean lattices) in the former approach. Thus, category theory tends to resolve logic in mathematics (into categories, on the one hand, and into functors or morphisms, on the other hand) leading the rethinking of logic started by its interpretation as Boolean algebra to a radical result after which the difference of logic and mathematics is rather due to the tradition and thus, conventional.

Furthermore, physical interpretations of category theory are also possible (and even emphasized by its creators as their motivation ${ }^{24}$ ): for example interpreting structures and categories as "bodies", and functors as "motions" or "changes" of those "bodies".

The idea of an eventual isomorphism suggested in the present section is underlain just by the interpretation of "morphisms" (or "functors") in category theory as transformation of tuples of axioms, each tuple of which is relevant to a certain category (rather than an extensional enumeration of all structures belonging to a certain category, and their mappings as morphisms or functors after that $)^{25}$. Then, any category (i.e. equivalent to a tuple of propositions, granted to be axioms, and thus obeying the rules of propositional logic) can be assigned unambiguously to a relevant interpretation of the "axes" of the qubit Hilbert space so that:

\footnotetext{
${ }^{24}$ For example, Eilenberg and Maclane (1945; in the introductory section of their first and fundamental paper on category theory) elucidate their idea by two dual vector spaces such as those utilized by quantum mechanics therefore suggesting or at least hinting at the approach in this section: the exhaustive interpretation of category theory by the qubit Hilbert space of quantum information. Furthermore, they emphasize expressively: "whenever new abstract objects are constructed in a specified way out of given ones, it is advisable to regard the construction of the corresponding induced mappings on these new objects as an integral part of their definition" (Eilenberg, Maclane 1945: 236). This declaration seems to be a translation (into a mathematical language) of the philosophical definition of "physical entity": changing itself by itself.

${ }^{25}$ Without any additional discussion (though necessary, but postponed for another paper), one can notice the possible conjecture that the double exchange of finiteness and infinity, on the one hand, and the intensional definitive property (or properties) of an infinite set and its extension consisting of an infinite number of elements, on the other hand, can be considered to be mutually conservable passing into each other by virtue of a certain common measure (e.g. such as probability). The former exchange is admissible in Hilbert arithmetic as that mediated by the dual Peano arithmetic, and the latter one, too, again mediated by the dual Peano arithmetic, but now relating to the pair of propositional logic and arithmetic (rather that to the pair of set theory and arithmetic as in the former case).
} 
1. An axis corresponds to an item in a certain axiomatic tuple.

2. The axiom at issue and its negation correspond to the pair of two qubits (respectively, "axes") idempotently dual to each other.

3. Any mathematical variable able to accept whether a finite or an infinite set of values can be represented equivalently as an axiomatic tuple correspondingly finite or infinite.

4. Mathematical variables assigning a single value are representable as values of a relevant qubit.

5. Logical variables being propositions assigning a binary value (i.e.: either "true" or "false") and can be represented by any pair of dual qubits,

6. Finally, the opposition of logical and mathematical variables is relative after the double exchange of logical and mathematical variables, on the one hand, and the global space consisting of bits with any local space of a single qubit.

As a conclusion, the qubit Hilbert space can be interpreted as the class of equivalence of all categories in category theory ${ }^{26}$, and thus, as a trivial isomorphism due to the identity to a class of equivalence in that sense is supplied.

\section{LEWIS CARROLL'S PARADOX BY HILBERT ARITHMETIC}

There exists the following problem about the relation of propositional logic, set theory, and arithmetic after they have been unified within the framework of Hilbert arithmetic as above. If set theory and propositional logic are consistent to each other (for example, after the Gödel (1930) completeness theorems and in that sense), but arithmetic and set theory are not (for example, after the Gödel (1931) incompleteness theorems), what is the relation of arithmetic and propositional logic? Is arithmetic consistent as a first-order logic to propositional logic? Can the Gödel dichotomy of arithmetic to set theory be transferred into the relation of propositional logic and arithmetic, therefore generating an analogical "either inconsistency, or incompleteness" of arithmetic to propositional logic, for example, as a first-order logic? Is possible an analogue (or even an isomorphic interpretation) of the Godel incompleteness theorems, in which set theory is substituted by propositional logic in virtue of sharing the same structure of Boolean algebra (though it being exhaustive for propositional logic, but only necessary for set theory needing furthermore two dual Peano arithmetics in the framework of Hilbert arithmetic)? Finally the last question (and most relevant to "What the Tortoise said to Achilles" interpreted by Hilbert arithmetic), does this import a kind of inconsistency in Hilbert arithmetic even if one grants that Hilbert arithmetic is free of Gödel's dichotomy?

Properly, "What the Tortoise said to Achilles" once interpreted in terms of Hilbert arithmetic brings the trouble and doubt emphasized by it. As if: the Gödel incompleteness is relevantly transposed within any implication therefore generating the ridiculous impossibility to be completed obviously contradicting common sense and human experience,

26 This is a rather philosophical consideration which corresponds to many proper mathematical approaches or methods for establishing "quantum category theory" (e.g. Rennela, Staton 2018; Moskaliuk, Moskaliuk 2013; Chikhladze 2011; Davis 2006; Holdsworth 1977, etc.). 
In fact, any implication in arithmetic as a first-order logic really cannot be accomplished directly: nonetheless, it can really be done absolutely consistently, but only in virtue of the consistency of propositional logic and set theory, on the one hand, and cut-elimination rule allowing the transition from an infinite series (respectively, set) of implication to a finite one, even to a single syllogism ${ }^{27}$. At that, cut-elimination is valid unambiguously only in that "direction": i.e. from an infinite series to a single syllogism, but not vice versa ${ }^{28}$ : no single syllogism implies unambiguously an infinite series of implications, due to which it needs new implications again and again as in Carroll's paradox ${ }^{29}$ : just that falsity of the statement (reverse to cut-elimination in a sense) is the subject of "What the Tortoise said to Achilles".

Speaking loosely, arithmetic by itself cannot be complete, and no implication can be accomplished being rigorously restricted to its framework just as the Tortoise stated or suggested: this is the analogue of the Gödel dichotomy ${ }^{30}$, but rethought in the relation of propositional logic and arithmetic (or respectively, it as a first-order logic). One needs set theory (though as a "Wittgenstein ladder") only after which any implication on;y within arithmetic can be consistently accomplished. Indeed, the dual copy of Peano arithmetic embedded in Hilbert arithmetic can be really always removed as a "Wittgenstein ladder" in virtue of the fact that the two copies are identical (though anti-isometric, but only as a relation to each other). Speaking again loosely, or in the Hegelian discourse intentionally emphasizing contradictions, Peano arithmetic just as "different from itself" (in Hegel's manner of expression) is set theory (i.e. by its dual counterpart identical to it in a sense).

27 Buss (2015) demonstrates how cut elimination can be applied to itself in a sense (i.e. as if self-referentially) allowing for removing all top-level cuts from proofs. Applying that method successively, one can reach a single syllogism finally.

${ }^{28}$ This statement can be also interpreted by means of Gentzen's cut elimination rule applied to Hilbert's $\varepsilon$-calculus (e.g. Wessels 1977) since the latter in turn can be reinterpreted by or as Hilbert arithmetic. Then, the sense of "non-identity" (respectively, "without identity") means just the "Dedekind set-theoretical finiteness" after which the mapping of an (or any) infinite set into infinite is possible only without identity, and that "non-identity" can be described rigorously by a probability density distribution unambiguously assignable to the finite-set mapping of the infinite set at issue. The characteristic function of that probability density distribution is a wave function, and thus, a transfinite natural number, respectively being a usual natural number, if it belongs to the dual Peano arithmetic. In the final analysis, the asymmetry of cut-elimination and "What the Tortoise said to Achilles" relies on the asymmetry of choice in the axiom of choice (or physically, on that of time arrow). In other words, if they were symmetric, this would imply reversible time, or set-theoretically, the idempotent exchangeability of an element of a set and the set itself, which is false even only in virtue of the availability of many elements belonging to the same set.

${ }^{29}$ For example, it can be represented by the relation of sequent calculi and cut elimination, that is, in the conceptual framework suggested and developed by Negri and Plato (2016).

${ }^{30}$ Horská (2014) suggests a kind of counter-analogy between the Gödel incompleteness versus the Gentzen completeness, which is absolutely relevant also in relation of the former to "What the Tortoise said to Achilles", on the one hand, and also, in relation of the latter to it, on the other hand. The Gödel incompleteness and the Gentzen completeness can be thought as a relation and its converse relation as to finiteness and infinity: the relation from infinity to finiteness (the Gentzen completeness) exists, but its converse, from finiteness to infinity does not exists and this is the sense of the Gödel incompleteness. 
Describing the same, the structure of Hilbert arithmetic substitutes the mystifying Hegelian discourse claiming the hypothetical "dialectic logic" in a way to be perfectly consistent to propositional logic (and thus, to the rational formal logic of syllogisms). A dual Peano arithmetic originating from the anti-isometric qubit Hilbert space is what one means as Peano arithmetic "different from itself" (said in the confusing Hegelian manner). Thus, that complementary counterpart supplies what one is to understand as the "non-arithmetic, but set-theoreic" in an explicit and constructive way. Then, just that "non-arithmetic, but set-theoretic" part is comprised by propositional logic to be consistent to set theory so that arithmetic (as set theory) is either incomplete or inconsistent to it, representing the essential "plot" of "What the Tortoise said to Achilles":

In other words or speaking loosely, Achilles can overtake the Tortoise both logically and physically only by means of the dual counterpart though the contest runs only in the initial Peano arithmetic and the dual "twin" can be even absolutely hidden in the ultimate result when Achilles has overtaken the Tortoise by any finite distance. ${ }^{31}$ The "Wittgenstein ladder" of the dual Peano arithmetic is necessary only in the infinitely small interval when Achilles is overtaking the Tortoise actually, but not after that, due to which it is removed.

The absolutely necessary mediation of the dual counterpart can be demonstrated negatively by the "Halting problem" of the Turing machine where the moment of overtaking is substituted by stopping the calculation, being mathematically isomorphic. Any Turing machine is definitively restricted to compute only in the framework of a single Peano arithmetic (conventionally called "initial" above): thus, it cannot calculate whether it will stop or not since it would need the mediation of a complementary Peano arithmetic (or Turing machine) rejected in default. Speaking figuratively, the insidious Tortoise forces Achilles to think as a Turing machine (therefore depriving him of human intellect).

The advantage of Hilbert arithmetic after the description of "What the Tortoise said to Achilles" consists just in the explicit referring to the dual Peano arithmetic, and then describing it in detail though it is not necessary within the ultimate result and can be removed in it as a "Wittgenstein ladder".

$X$ INSTEAD OF CONCLUSION: THE UNITY OF LOGIC, PHYSICS AND MATHEMATICS BEING COMPLETE

The necessary unification of physics, mathematics, and philosophy once Hilbert arithmetic has been introduced is discussed in a few papers (e.g.: Penchev 2021 July 26; 2021 August 24; 2021 November 18). Now, the specific accents are two: (1) logic by means of propositional logic in the framework of that unity; (2) completeness as the sufficient condition for the unity.

The contemporary realization of logic seems to be "forked" into specific mathematical structures of categories (such as lattices), on the one hand, or non-classical logics, on the other hand, modifying in a way or another the traditional formal logic of syllogisms. That bifurcation

${ }^{31}$ The same can be represented rather intuitively following the ideas of White (1999) about the relation of simulation of a physical object and a relevant deduction in the framework of its mathematical model especially to cut-elimination. 
can be noticed still when Boolean algebra appeared being both a mathematical structure (an algebra) and simultaneously, granted to be equivalent to the logic of syllogisms. (Carroll's paradoxes are interpreted to be devoted to the troubles of that eventual equivalence). If logic is understood as a share of mathematics (i.e. in the former branch being historically second), one does not need to debate separately its belonging to the unity in question. However, logic as a collection of non-classical logics changing ones or other axioms of classical logic needs a special consideration to be added in the scope of the unity.

Just "What the Tortoise said to Achilles" centered into the problems of distinguishing Boolean algebra and propositional logic by syllogisms is quite relevant to the discussion. The Hegelian opposition of formal and "dialectic logic", the latter in turn granted to be the proper "ontological logic" including that of the physical world and mechanical motion, is a natural complementing background of the same problem; even more so that many "paraconsistent logics" claiming to formalize, and thus, to originate directly from dialectic logic, have been created and belong to the scope of "non-classical logic".

As far as any non-classical logic proves its consistency by a model in classical logic, one can restrict the discussion only to the traditional syllogistic logic. If it is granted to be equivalent to Boolean algebra, the problem is resolved. So, the question asked by "What the Tortoise said to Achilles" is essential also about eventual obstacles in the pathway for logic to be thoroughly integrated with physics (after mathematics by the category of lattice) and thus with philosophy following Hegel's objective, but by formal and logical tools.

Properly, the problem of the paradox (especially emphasized by Carroll by means of the allusion to Zeno's one) is about whether or how infinity is admissible in logic and whether that problem (about the applicability of infinity in logic) does not distinguish Boolean algebra (where infinity seems to be relevant as in any mathematical structure) from classical logic referring to successive syllogisms: where an infinite series of syllogisms seems to be inaccessible whether to the finite human mind or as a process that takes place over time ${ }^{32}$ (and thus any infinite syllogism turns out to be never finished, moreover, each single implication entails an infinite syllogism).

Furthermore, the same opposition of infinity to finiteness is subject of the Gödel incompleteness theorems implying his dichotomy. In fact, Lewis Carroll's antinomy anticipates them, since it shares the same dichotomy however exemplified by a special set of syllogisms (that of an iterative series of syllogisms). Classical logic calls the natural numbers of arithmetic to enumerate those syllogisms as successive to each other therefore implying for their number to be always finite in virtue of the axiom of induction.

On the contrary, Boolean algebra being a mathematical structure is situated on the side of set theory and infinity, naturally admitting an infinite set of propositions thus enumerable by the set of all natural numbers. Then, one can repeat literally the Gödel dichotomy, this time in relation to syllogistic logic and Boolean algebra:

\footnotetext{
${ }^{32}$ Valaris (2017) justifies "that there are diachronic norms of epistemic rationality - namely, norms of good reasoning" (the author's italic) meaning a necessary mental jump from the premises to the conclusion corresponding to different ("diachronic") moments of time.
} 
Syllogistic logic is either incomplete or inconsistent to Boolean algebra: and this statement can be immediately visualized by "What the Tortoise said to Achilles", after which even any implication cannot be accomplished since it is equivalent to an infinite set of syllogisms, which contains at least one neither true nor false. The paradox is "true", and this means that the completeness of syllogistic logic to Boolean algebra (though both are granted by common sense to be equivalent to each other) is just so unprovable as arithmetic to set theory under the crucial condition of "Gödel mathematics". That is: it is provable, but in "Hilbert mathematics":

Hilbert arithmetic in turn reforms and unifies consistently Peano arithmetic, set theory, and propositional logic in a way they to be the reliable foundations of Hilbert mathematics, only after which Carroll's paradox about Achilles and the Tortoise can be resolved rigorously and logically for it has been rooted in the Gödel dichotomy, which is to be removed before that.

Once Hilbert mathematics is established, all non-classical logics grounded in relevant modified tuples of the axioms of classical logic can be reconciled consistently with their interpretations as mathematical structure (for example belonging to the category of lattices).

What remains to be justified is "(2)" above: how the concept of completeness assists or necessary implies (from a proper logical and mathematical viewpoint) the unity of logic, mathematics, physics, and philosophy:

As to philosophy, the idea of Kant's transcendentalism embodies completeness (rather postulated by the concept of the totality) as a necessary basis for Cartesian dichotomy and dualism to be resolved monistically: anyway it is a metaphysical (in a Popper sense) and thus, a fundamentally unfalsifiable statement as a philosophical doctrine therefore needing a relevant scientific reformulation to be reusable in exact and deductive sciences such as mathematics and logic or even in an experimental science such as physics. This is its counterpart of "scientific transcendentalism" discussed in detail in other papers (e.g. Penchev 2020 October 20).

As to physics (ibid.), completeness can be revealed in both concepts of the universe and the proved completeness of quantum mechanics once it has been grounded into the separable complex Hilbert space. Just the latter is the relevant bridge to completeness in mathematics, however fundamentally impossible in the framework of "Gödel mathematics".

Then only Hilbert mathematics based on Hilbert arithmetic is able to embody consistently completeness: at that, following the analogical or isomorphic approach and formal structure established by quantum mechanics. The last touch of completeness, as to logic, can be painted investigating Lewis Carroll's "What the Tortoise said to Achilles", by which one can ascertain that the completeness of logic though often granted to be self-evident, in fact is so problematic as that of mathematics, but fortunately even isomorphic. Thus, once a solution is revealed in mathematics, it can be merely repeated in logic in virtue of that isomorphism:

Hilbert arithmetic underlies rigorously and reliably the unity and completeness of logic, mathematics, physics, and philosophy. 


\section{REFERENCES:}

Aerts, D., J. Broekaert, S. Smets (1999) "A Quantum Structure Description of the Liar Paradox," International Journal of Theoretical Physics 38 (12): 3232-3239.

Belkind, O. (2018) "In defense of Newtonian induction: Hume's problem of induction and the universalization of primary qualities," European Journal for Philosophy of Science 9 (1): 13-26.

Brown, D. (1954) "What the Tortoise taught us," Mind LXIII (250): 170-179.

Burks, A. W., Copi, I. W (1950) “Lewis Carroll's Barber Shop Paradox,” Mind LIX (234): 219-222.

Buss, S. (2015) "Cut elimination in situ," in: (Kahle, R. and M. Rathjen, eds.) Gentzen's Centenary (Chapter 10) Cham, Switzerland: Springer Switzerland, pp. 245-277.

Conway, J. and S. Kochen (2006) "The Free Will Theorem," Foundations of Physics 36 (10): 1441-1473.

Conway, J. and S. Kochen (2009) "The Strong Free Will Theorem," Notices of the AMS 56 (2): 226-232.

Carroll, L. (1894) "A Logical Paradox," Mind 3 (11): 436-438.

Carroll, L. (1895) "What the Tortoise said to Achilles," Mind 4 (14): 278-280 (reprinted in 1995 in: Mind 104 (416): 691-693).

Chikhladze, D. (2011) "Elements of enriched and quantum category theory," Bulletin of the Australian Mathematical Society 83 (3): 518-519.

Davis, S. (2006) "Quantum Theory and the Category of Complex Numbers," International Journal of Theoretical Physics 45 (10): 1914-1929.

Diamond, G. A., \& Kaul, S. (2010) "What the Tortoise Said to Achilles," The American Journal of Cardiology 106 (4): 593-595.

Eilenberg, S.; S. MacLane (1945) "General Theory of Natural Equivalences," Transactions of the American Mathematical Society 58 (2): 231-294

Fumerton, R. (2015) "What the Internalist should say to the Tortoise," Episteme 12 (Special Issue 2): 209-217.

Gentzen, G. (1935) "Untersuchungen über das logische Schließen. I," Mathematische Zeitschrift 39 (2): 176-210.

Gentzen, G. (1935) "Untersuchungen über das logische Schließen. II," Mathematische Zeitschrift 39 (3): 405-431.

Giandomenico, S., ed. (2006) What Is Category Theory? (Advanced Studies in Mathematics and Logic 3). Monza, Italy: Polimetrica.

Gillon, G.G. (1997) "Contraposition and Lewis Carroll's Barber Shop Paradox," Dialogue 36 (2): 247 252.

Hawson, C. (2000) Hume's Problem: Induction and the Justification of Belief. Oxford - New York: Clarendon Press.

Holdsworth, D.G. (1977) "Category theory and quantum mechanics (kinematics)," Journal of Philosophical Logic 6 (1): 441-453.

Horská, A. (2014) Where is the Gödel-Point Hiding: Gentzen's Consistency Proof of 1936 and his Representation of Constructive Ordinals (Springer Briefs in Philosophy). Cham, Switzerland: Springer

Jackson, A. (2019) "How to solve Hume's problem of induction," Episteme 16 (2): 157-174.

Jacobson, A. J. (1987) "The problem of induction: what is Hume's argument?" Pacific Philosophical Quarterly 68 (3-4): 265-284.

Kochen, S., E. P. Specker (1967). "The problem of hidden variables in quantum mechanics," Journal of Mathematics and Mechanics 17 (1): 59-87.

Krömer, R. (2007) Tool and Object: A History and Philosophy of Category Theory (Science Networks. Historical Studies). Basel: Birkhäuser. 
Kuś, M. and B. Skowron, eds. (2019) Category Theory in Physics, Mathematics, and Philosophy (Springer Proceedings in Physics: Volume 235), Cham, Switzerland: Springer Nature Switzerland.

Lange, M. (2011) "Hume and the Problem of Induction," in: (D. M. Gabbay, S. Hartmann, J. Woods, eds.) Inductive Logic (vol. 10 of "Handbook of the History of Logic"). Amsterdam, etc.: North Holland - Elsevier, pp. 43-91.

Lantin, R. (1998) "Hume and the problem of induction," Philosophia 26 (1-2) 105-117.

Laraudogoitia, J. P. (2014) "What the Tortoise Said to Achilles," Philosophia 42 (2): 405-411.

Lipton, P. (2002) "Colin Howson, Hume's Problem: Induction and the Justification of Belief," The British Journal for the Philosophy of Science 53 (4): 579-583.

Marquis, J.-P. (2009) From a Geometrical Point of View: A Study of the History and Philosophy of Category Theory (Logic, Epistemology, and the Unity of Science; 14). New York: Springer.

Moctefi, A., F.F. Abeles, eds. (2016) "'What the Tortoise Said to Achilles': Lewis Carroll's Paradox of Inference," The Carrollian: The Lewis Carroll Journal 28 (special double issue).

Moskaliuk, N. M. and S.S. Moskaliuk (2013) "Category of trees in representation theory of quantum algebras," Physics of Atomic Nuclei 76 (10): 1257-1267

Negri, S. and J. von Plato (2016) "Cut Elimination in Sequent Calculi with Implicit Contraction, with a Conjecture on the Origin of Gentzen's Altitude Line Construction," in (Probst, D. and P. Schuster, eds) Concepts of Proof in Mathematics, Philosophy, and Computer Science. Berlin - Boston: De Gruyter.

von Neumann, J. (1932) Grundlagen der Quantenmechanik. Berlin: Springer, pp. 167-173.

Otero, M. P. (2008) "The Humean problem of induction and Carroll's Paradox," Philosophical Studies 141 (3): 357-376.

Penchev, V. (2021 November 18) “'Noema' and 'Noesis' by Information after Husserl's Phenomenology Interpreted Formally," SSRN, $\quad \underline{\text { https://dx.doi.org/10.2139/ssrn.3943811 }}$ or https://papers.ssrn.com/sol3/papers.cfm?abstract id=3943811.

Penchev, V. (2021 August 24) "Hilbert arithmetic as a Pythagorean arithmetic: arithmetic as transcendental," SSRN, https://papers.ssrn.com/sol3/papers.cfm?abstract id=3909610 or https://dx.doi.org/10.2139/ssrn.3909610.

Penchev, V. (2021 July 26) "Quantum phenomenology as a "rigorous science": the triad of epoché and the symmetries of information," SSRN, https://papers.ssrn.com/sol3/papers.cfm?abstract id=3892039 https://dx.doi.org/10.2139/ssrn.3892039.

Penchev, V. (2021 April 12) "Both Classical \& Quantum Information; Both Bit \& Qubit: Transcendental Time. Both Physical \& Transcendental Time," SSRN, https://dx.doi.org/10.2139/ssrn.3823665 , https://papers.ssrn.com/sol3/papers.cfm?abstract id=3823665 .

Penchev, V. (2021 February 25) "Natural Cybernetics of Time, or about the Half of any Whole," SSRN, https://papers.ssrn.com/sol3/papers.cfm?abstract id $=3750608$ or

https://dx.doi.org/10.2139/ssrn.3750608.

Penchev, V. (2020 December 14) "Natural Cybernetics and Mathematical History: The Principle of Least Choice in History," SSRN, https://papers.ssrn.com/sol3/papers.cfm?abstract id=3714119 or https://dx.doi.org/10.2139/ssrn.3714119.

Penchev, V. (2020 October 20) "Two deductions: (1) from the totality to quantum information conservation; (2) from the latter to dark matter and dark energy," SSRN, https://papers.ssrn.com/sol3/papers.cfm?abstract id=3683658, https://dx.doi.org/10.2139/ssrn.3683658 .

Penchev, V. (2020 August 25)“The Relationship of Arithmetic As Two Twin Peano Arithmetic(s) and Set Theory: A New Glance From the Theory of Information," SSRN, https://dx.doi.org/10.2139/ssrn.3656179, https://papers.ssrn.com/sol3/papers.cfm?abstract id=3656179. 
Penchev, V. (2020 July 24) "Physical Entity As Quantum Information." SSRN, https://papers.ssrn.com/sol3/papers.cfm?abstract id=3620702, https://dx.doi.org/10.2139/ssrn.3620702 .

Penchev, V. (2020 July 16) "If Quantum Mechanics Is the Solution, What Should the Problem Be?" SSRN, https://papers.ssrn.com/sol3/papers.cfm?abstract id $=3633784$ or https://dx.doi.org/10.2139/ssrn.3633784 .

Penchev, V. (2020 July 10) "Quantum information as the information of infinite collections or series," SSRN, https://papers.ssrn.com/sol3/papers.cfm?abstract id $=3630063$ or https://dx.doi.org/10.2139/ssrn.3630063 .

Penchev, V. (2010) "Insolvability of the first incompleteness theorem. Gödel and Hilbert mathematics," Philosophical Alternatives 19 (5): 104-119 (in Bulgarian: Пенчев, В. "Неразрешимост на първата теорема за непълнотата. Гьоделова и Хилбертова математика,” Философски алтернативи).

Penchev, V. (2009) Philosophy of Quantum Information. Volume 1. Einstein and Gödel. Sofia: IPhR BAS (in Bulgarian: В. Пенчев Философия на квантовата информаџия. Том 1. Айнщайн и Гьодел София: ИФИ - БАН).

Peruzzi, A. (2006) "The Meaning of Category Theory for 21st Century Philosophy," Axiomathes 16 (4): 424-459.

Rees, W.J. (1951) "What Achilles said to the Tortoise," Mind LX (238): 241-246.

Rennela, M. and S. Staton (2018) "Classical Control and Quantum Circuits in Enriched Category Theory," Electronic Notes in Theoretical Computer Science 336 (4): 257-279.

Roy, S.M. (2001) “Quantum Zeno and anti-Zeno paradoxes,” Pramana 56 (2-3): 169-178.

Robinson, A. (1966) Non-Standard Analysis. Amsterdam: North-Holland Publishing Company.

Schueler, G. F. (1995) Why "Oughts" are not Facts (or What the Tortoise and Achilles Taught Mrs. Ganderhoot and Me about Practical Reason)," Mind (New Series) 104 (416): 713-723.

Skolem, T, (1922) "Einige Bemerkungen zur axiomatischen Begründung der Mengenlehre," in: Matematikerkongressen i Helsingrofs den 4-7 Juli 1922, Den femte skandinaviska matematikerkongressen. Redogörelse, Helsinki: Akademiska-Bokhandeln, pp. 217-232 (1923)

Tucker, C. (2009) "Evidential Support, Reliability, and Hume's Problem of Induction," Pacific Philosophical Quarterly 90 (4): 503-519.

Valaris, M. (2017). "What The Tortoise Has To Say About Diachronic Rationality," Pacific Philosophical Quarterly 98 (S1): 293-307.

Weintraub, R. (1995) "What Was Hume's Contribution to the Problem of Induction?"

The Philosophical Quarterly 45 (181): 460-470.

Weintraub, R. (2008) “A Problem for Hume's Theory of Induction,” Hume Studies 34 (2): 169-187.

Wessels, L. (1977) "Cut Elimination in a Gentzen-Style $\epsilon$-Calculus without Identity," Mathematical Logic Quarterly 23 (36): 527-538.

White, G.G. (1999) "Simulation, Theory, and Cut Elimination," The Monist 82 (1: "Philosophy of Computer Science"): 165-184.

Wright, S. (2015). "Comments on "What the Internalist Should Say to the Tortoise," Episteme 12 (2): 219-223. 